\title{
Magnetic Edge Anisotropy in Graphenelike Honeycomb Crystals
}

\author{
J. L. Lado and J. Fernández-Rossier \\ International Iberian Nanotechnology Laboratory (INL), Avenida Mestre José Veiga, 4715-330 Braga, Portugal
}

(Received 18 February 2014; revised manuscript received 16 May 2014; published 10 July 2014)

\begin{abstract}
The independent predictions of edge ferromagnetism and the quantum spin Hall phase in graphene have inspired the quest of other two-dimensional honeycomb systems, such as silicene, germanene, stanene, iridates, and organometallic lattices, as well as artificial superlattices, all of them with electronic properties analogous to those of graphene, but a larger spin-orbit coupling. Here, we study the interplay of ferromagnetic order and spin-orbit interactions at the zigzag edges of these graphenelike systems. We find an in-plane magnetic anisotropy that opens a gap in the otherwise conducting edge channels that should result in large changes of electronic properties upon rotation of the magnetization.
\end{abstract}

DOI: 10.1103/PhysRevLett.113.027203

PACS numbers: 75.30.Gw, 71.30.+h, 75.70.Rf

Magnetic anisotropy, a technologically crucial property, is driven by spin-orbit interaction, which is normally the underdog in the competition with the other two terms that control ferromagnetism, namely, kinetic and Coulomb energy [1]. As a result, magnetic anisotropy energy in conventional ferromagnets is at least 2 orders of magnitude smaller than the Curie temperature and the Fermi energies (or the band gap, in the case of insulators). For the same reason, transport properties in ferromagnetic metals are only weakly dependent on the magnetic orientation, and typical values for the anisotropic magnetoresistance are below $3 \%$ [1].

Here, we study magnetic anisotropy in a class of systems for which the balance between these three energy scales is very different from the usual, which leads to two dramatic consequences, very different from conventional ferromagnetism. First, the conducting properties change from metal to insulator, depending on the magnetization orientation, an effect that, to the best of our knowledge, has never been reported. Second, the magnetic moment magnitude depends strongly on the magnetic orientation, and it can change or even vanish in some directions, a phenomenon dubbed colossal magnetic anisotropy [2,3]. The class of systems in question is the zigzag edges of two-dimensional honeycomb crystals [4] whose electronic properties can be described with a tight-binding model with a single orbital per site and Kane-Mele spin-orbit interactions [5]. This includes several materials, such as group IV two-dimensional crystals (graphene [5], silicene [6-8], germanene [9], and stanene [10]), the double layer perovskite iridates [11,12], and metal organic frameworks (MOFs) [13]. In addition, given that the existence of nondispersive edge states occurs at the zigzag edge of any system described with the Dirac equation [14], the results discussed here should also be valid for the so-called designer Dirac fermions formed in "artificial graphene" formed by the decoration of two-dimensional electron gases with honeycomb arrangements $[15,16]$.
Ignoring spin-orbit and Coulomb interactions altogether, these 2D crystals are zero band-gap semiconductors with Dirac-like dispersion close to the Fermi energy. Zigzag edges in these systems are known to host localized edge states that, when both Coulomb and spin-orbit coupling are neglected, are nondispersive, sublattice polarized, and lie precisely at the Fermi energy, at half-filling [4]. The ensuing large density of states results in a Stoner instability that leads to ferromagnetic order at the edge [17-20].

On the other hand, Kane-Mele spin-orbit interaction, a second-neighbor spin-dependent hopping that conserves the spin component $s_{z}$ perpendicular to the two-dimensional crystal [5], has dramatic consequences in these honeycomb crystals. It opens a topologically nontrivial gap in bulk and the emergence of in-gap spin-filtered dispersive edge states: for a given spin projection $s_{z}$, electrons propagate along one direction only, preventing backscattering even in the presence of time-reversal symmetric disorder. Importantly, the slope of the edge bands is proportional to the Kane-Mele spin-orbit coupling, which controls thereby the density of states at the Fermi energy. The interplay of spin-orbit and Coulomb repulsion on the otherwise nondispersive edge states leads to the strong magnetic anisotropy effects anticipated above.

To model this kind of system, we use the so-called KaneMele-Hubbard Hamiltonian [21,22], which provides a minimal model to study the effect of the Coulomb interactions on the topologically protected edge states:

$$
H=\sum_{\langle i j\rangle, \sigma} t c_{i \sigma}^{\dagger} c_{j \sigma}+\sum_{\langle\langle i j\rangle\rangle, \sigma} i t_{\mathrm{SO}} \sigma \nu_{i j} c_{i \sigma}^{\dagger} c_{j \sigma}+H_{\mathrm{int}},
$$

where $\sigma= \pm 1$ are the spin projections of the spin along the axis perpendicular to the two-dimensional crystal, the single angled brackets stand for first neighbor and double angled brackets for second, and $\nu_{i j}= \pm 1$ for clockwise or anticlockwise second-neighbor hopping [5,23]. 
For simplicity, we neglect the Rashba coupling [24,25]. In the case of planar honeycomb systems, such as graphene, the Rashba term is null. For buckled group IV crystals, such as silicene, germanene, and stanene, the magnitude of the Rashba is 1 order of magnitude lower than the pure spin orbit [26].

The Hubbard term reads

$$
H_{\mathrm{int}}=U \sum_{i} n_{i \uparrow} n_{i \downarrow}
$$

where $n_{i \uparrow}=c_{i \uparrow}^{\dagger} c_{i \uparrow}$ denotes the occupation operator of site $i$ with spin $\uparrow$ along an arbitrary quantization axis. We treat the Hubbard interaction in the collinear mean field approximation, enforcing the magnetization to lie along the axis $\vec{\Omega}=(\sin \alpha, 0, \cos \alpha)$ that we take as the quantization axis [see Fig. 2(a)]. This approach permits us to study solutions with different $\alpha$ and compare their properties. Rotations in the $x y$ plane leave the results invariant, due to the symmetry of the Kane-Mele spin-orbit coupling. In general, the Coulomb interaction term evaluated in the mean field approximation leads to two self-consistent potential terms, direct and exchange. In the case of the Hubbard model in the collinear approximation, only the direct term survives:

$H_{\mathrm{MF}}=U\left[\left\langle n_{i \uparrow(\alpha)}\right\rangle n_{i \downarrow(\alpha)}+n_{i \uparrow(\alpha)}\left\langle n_{i \downarrow(\alpha)}\right\rangle-\left\langle n_{i \uparrow(\alpha)}\right\rangle\left\langle n_{i \downarrow(\alpha)}\right\rangle\right]$,

where the notation explicitly shows that the spin quantization axis is taken along $\vec{\Omega}(\alpha)$ and $\left\langle n_{i \uparrow(\alpha)}\right\rangle$ stand for the average of the occupation operator calculated within the ground state of the mean field Hamiltonian:

$$
H=H_{0}+H_{\mathrm{MF}} .
$$

As usual, this defines a self-consistent problem that we solve by iteration. Because of the spin-orbit Kane-Mele term in $H_{0}$, mean field solutions with different $\alpha$ are not equivalent. Notice as well that the $H_{\mathrm{MF}}$ term is nondiagonal when represented in the basis of eigenstates of $S_{z}$ and $\alpha \neq 0$ [27].

We pay special attention to the atomic magnetization, along the $\vec{\Omega}(\alpha)$ in site $i$ :

$$
m_{i(\alpha)}=g \mu_{B} \frac{\left[\left\langle n_{i \uparrow(\alpha)}\right\rangle-\left\langle n_{i \downarrow(\alpha)}\right\rangle\right]}{2},
$$

and we take $g=2$.

In order to study the zigzag edges, it is convenient to study ribbons that define a one-dimensional crystal (see Fig. 1) with two edges. A given unit cell of the onedimensional crystal is formed by $N$ units of four atoms. In the following, we characterize the width of the ribbons by $N$. For finite $U$, and as long as $t_{\mathrm{SO}} / U$ is not too large, we find solutions with ferromagnetic order at the edges. The magnetic moment calculated self-consistently is
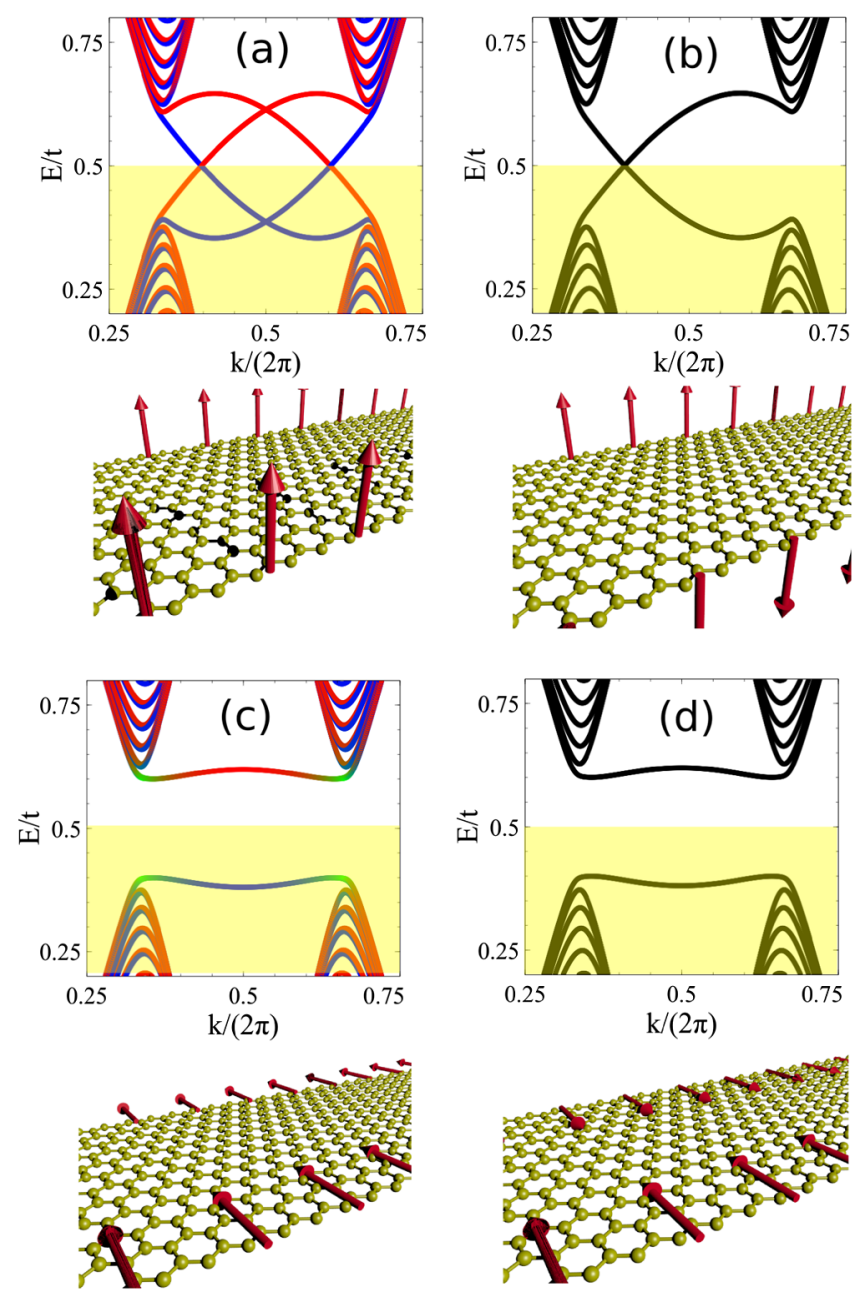

FIG. 1 (color online). Four different ferromagnetic configurations, either in or off plane and parallel (FM) or antiparallel (AF) edge magnetization, together with their band structures calculated within the mean field Kane-Mele-Hubbard model. (a),(b) Off plane and conducting (both for FM and AF arrangements). (c),(d) In-plane and insulating parallel (both FM and AF) magnetizations. Calculations are done with $U=t$ and $t_{\mathrm{SO}}=0.02 t$.

non-negligible only at the edge atoms. Attending to their mutual magnetization orientation, ribbons yield two types of solutions with ferromagnetic edges: parallel [ferromagnetic (FM)] and antiparallel [antiferromagnetic (AF)]. For sufficiently wide ribbons, the interedge coupling is negligible and both solutions have identical properties.

The first important result of the paper is shown in Fig. 1. Whereas off-plane magnetization $(\alpha=0)$ leads to a conducting solution, found in previous works [22], the in-plane magnetization opens a gap. Therefore, transport properties of zigzag edges will change dramatically upon rotation of the magnetization direction, in contrast with conventional metallic ferromagnets. This metal-insulator transition is developed as well in chiral edge ribbons [27], which have been widely reported $[33,34]$. 
The second important result of the manuscript is shown in Fig. 2(b). The ground state energy $E(\alpha)$ is minimal for $\alpha=\pi / 2$, i.e., for in-plane magnetization, which means that spontaneous magnetic order in this system leads to insulating behavior.

The results of Fig. 1 can be understood as follows. In the absence of magnetic order, two spin-filtered in-gap edge states with opposite velocities exist at each edge [5], resulting in a twofold degeneracy (not shown). Ferromagnetic order with $\vec{\Omega}=\hat{z}$ breaks time-reversal symmetry but does not mix spins. Thus, magnetic order merely yields a spin-dependent shift that breaks the twofold degeneracy, as seen in Fig. 1(a), for the FM case. For the AF configuration, there is an extra symmetry that restores the double degeneracy: the combined action of spatial inversion, which results in an exchange of the atoms of the two interpenetrating triangular sublattices $A$ and $B$ that form the honeycomb, and time reversal, which exchanges $\uparrow$
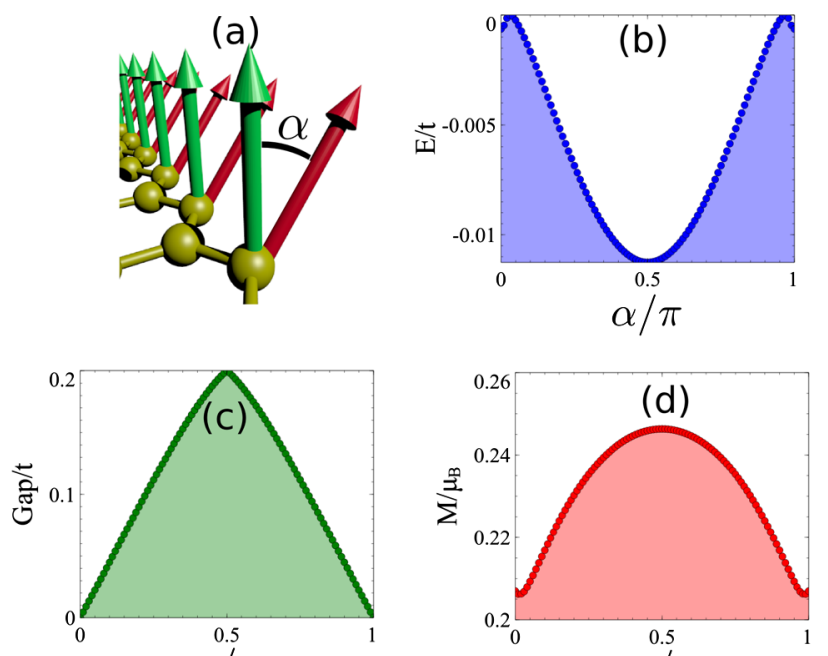

(e)
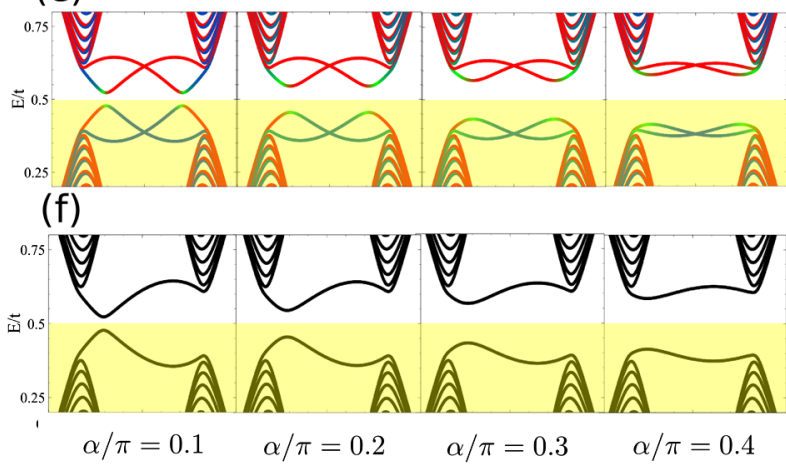

FIG. 2 (color online). Evolution of electronic properties for the FM ribbon as a function of the magnetization direction $\vec{\Omega}=(\sin \alpha, 0, \cos \alpha)$. (a) Scheme of the edge magnetization for two different angles 0 and $\alpha$. Ground state (b) total energy (per unit cell, with two magnetic atoms per cell), (c) gap, and (d) magnetization as a function of $\alpha$. (e),(f) Evolution of the band structure for different values of $\alpha$ for the (e) FM and (f) AF configurations. $N=30, U=t$, and $t_{\mathrm{SO}}=0.02 t$.
TABLE I. Energy scales for different graphenelike honeycomb materials.

\begin{tabular}{lccc}
\hline \hline Material & $t_{\text {SO }}$ & $t$ & Reference \\
\hline Graphene & $0.1-5.0 \mu \mathrm{eV}$ & $2.7 \mathrm{eV}$ & {$[29-31]$} \\
Silicene & $0.16 \mathrm{meV}$ & $1.5 \mathrm{eV}$ & {$[26,32]$} \\
Germanene & $2.5 \mathrm{meV}$ & $1.4 \mathrm{eV}$ & {$[26,32]$} \\
Stanene & $8-30 \mathrm{meV}$ & $1.3 \mathrm{eV}$ & {$[10,26]$} \\
MOF & $1 \mathrm{meV}$ & $0.3 \mathrm{eV}$ & {$[13]$} \\
Double perovskite & $1 \mathrm{meV}$ & $0.1 \mathrm{eV}$ & {$[12]$} \\
\hline \hline
\end{tabular}

and $\downarrow$, leaves the system invariant. Thus, the spin $\uparrow$ band localized at the $A$-type edge is degenerate with the spin $\downarrow$ band localized in the opposite edge.

The situation is radically different when the magnetization lies in plane. Representing the self-content potential in the basis of the $U=0$ spin-filtered edge states, with spin quantized along the $\hat{z}$ axis, the effect of the in-plane magnetization is to mix bands with opposite spins. As a result, a band gap opens at the $k$ point where the noninteracting edge bands cross. The evolution of the bands as the magnetization is rotated from almost off plane (left) to almost in plane (right) is shown in Figs. 2(e) and 2(f). It is apparent that the band gap [Fig. 2(c)] is maximal for inplane magnetization $(\alpha=\pi / 2)$ and null for off plane $\alpha=0$. The preference for in-plane magnetization can also be connected with the variation of the magnitude of the edge magnetic moment with $\alpha$ [Fig. 2(d)]. These two results naturally explain the fact that the ground state energy is minimal for in-plane magnetization. At halffilling, all the valence bands are occupied and the conduction bands are empty. Therefore, increasing the band gap decreases the total energy.

The gap opening as long as magnetization is not off plane will certainly have dramatic consequences on the transport properties along the edges. A result similar to this has been obtained recently [35], using a Kane-Mele model where magnetic order is externally driven and modeled by a magnetic exchange potential that arises from proximity rather than spontaneity, as discussed here.

The results of Figs. 1 and 2 are, for a specific choice of $U / t=1$ and $t_{\mathrm{SO}} / t=0.02$, and, for a ribbon with $N=30$ sites, wide enough to decouple the two edges. We now discuss how the results depend, quantitatively, on the specific values of the spin-orbit coupling, ribbon width, and $U$. The evolution of several energy differences between $\mathrm{AF}$ and FM and in-plane-off-plane configurations, as a function of the ribbon width $N$, is shown in Fig. 3(a). For large $N$, it is apparent that FM and AF have the same ground state and anisotropy energy. In addition, the edge gap [Fig. 3(b)] also becomes independent on the magnetic configuration at large width.

In Figs. 3(c) and 3(e), we plot the dependence of the magnetic anisotropy and magnetic moment (both in and off plane) on the magnitude of the spin-orbit coupling $t_{\mathrm{SO}} / t$, 

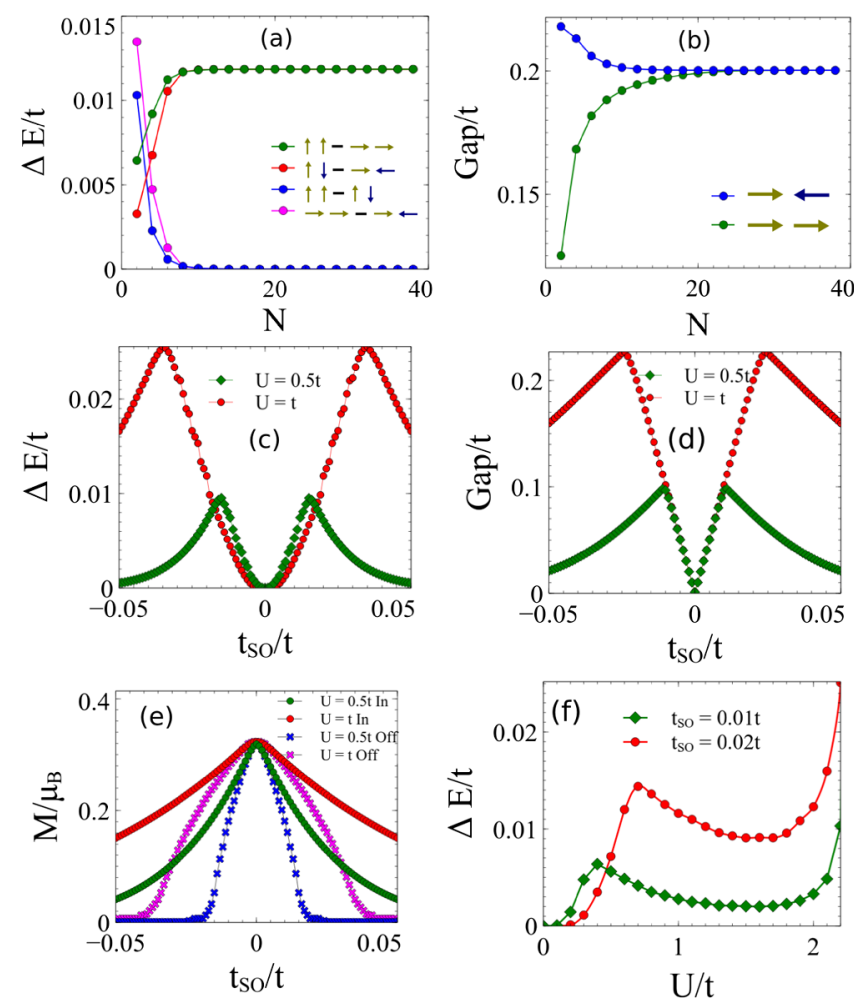

FIG. 3 (color online). (a) Evolution of the energy differences between the in-plane vs off-plane as well as the FM vs AF configurations (four cases) as a function of ribbon width $N$. (b) Gap, for the in-plane magnetization solution, for FM and AF solutions, as a function of $N$. (c)-(e) Evolution with the strength of the spin-orbit coupling $t_{\mathrm{SO}}$ : (c) anisotropy energy, (d) gap, and (e) edge magnetization. (f) Anisotropy energy with the on-site Hubbard interaction. In (a) and (b), $U=t$ and $t_{\mathrm{SO}}=0.02 t$. In (c) and (d), $N=30$.

for two different values of $U$. Attending to the difference between the magnitude of the magnetic moment in the offplane and in-plane cases [Fig. 3(e)], three different regions are found. For very small $t_{\mathrm{SO}} / t$, the magnetic moment is the same for out-of-plane and in-plane magnetization and the magnetic anisotropy energy depends quadratically on $t_{\mathrm{SO}} / t$. From this standpoint, the behavior of the zigzag edge is similar to conventional magnets, although it is a small gap, open for in-plane magnetization. For wide enough ribbons, the value of this gap is given by $\min \left(\Delta_{2 \mathrm{D}}, U m_{\text {edge }}\right)$, where $\Delta_{2 \mathrm{D}}=6 \sqrt{3} t_{\mathrm{SO}}$ is the bulk gap opened by spin-orbit interaction and $U m_{\text {edge }}$ is the exchange splitting gap, which is a decreasing function of $t_{\text {SO }}$, giving rise to the curve seen in Fig. 3(d).

For intermediate values of $t_{\mathrm{SO}} / t$, it is apparent that the magnetic moment magnitude is different for in-plane and off-plane orientations, but in both cases, it is finite. In this region, the anisotropy energy scales approximately linearly with $t_{\mathrm{SO}} / t$ and the band gap of in-plane magnetization is still a linear function of $t_{\mathrm{SO}} / t$. Finally, above a given critical value $t_{\mathrm{SO}} \simeq 0.02 t(0.04 t)$ for $U=0.5 t(U=t)$, the system enters in the so-called colossal [2,3] magnetic anisotropy regime, for which magnetic order is only possible in plane and the magnetic solutions off plane do not exist. Increasing spin-orbit coupling beyond this point starts to reduce the band gap and the magnetic order altogether, which leads to a reduction of the magnetic anisotropy energy [Fig. 3(e)].

We thereby expect that graphene, silicene, and germanene are in the small $t_{\mathrm{SO}} / t$ region, the MOF is in the intermediate region, and the stanene zigzag edge could show the colossal magnetic anisotropy effect (see Table I). Notice that in the intermediate region, the magnetic anisotropy energy per magnetic atom can be extremely large. For instance, for stanene, taking $U=t$, intermediate $t_{\mathrm{SO}} \simeq 8 \mathrm{meV}$, and $t \simeq 1.3 \mathrm{eV}$, we obtain $\Delta E \simeq 4 \mathrm{meV}$, significantly larger than record materials such as $\mathrm{YCo}_{5}$ [1]. $\mathrm{Na}_{2} \mathrm{IrO}_{3}$ and related systems [11,12] also offer a fascinating possibility of real tuning of the effective $t_{\mathrm{SO}}$ by strain [12], which would make it possible to build devices with straintunable anisotropy.

Given the spread of estimates of the actual values of $U$ for a given material, as well as the fact that different substrates can result in different values of $U$, we address the question of how the results above depend on the strength of the on-site Coulomb repulsion $U$. At finite $t_{\mathrm{SO}}$, there is a critical $U_{c 1}$ below which the edges are nonmagnetic [22] and a second $U_{c 2}$ above which the entire honeycomb lattice becomes antiferromagnetic [17]. For $U_{c 1}<U<U_{c 2}$, only the edge is magnetic, and its magnetic anisotropy energy is a nonmonotonic function of $U$. It increases first, reflecting the increase of the magnetic moment, and then it decreases slightly, reflecting the reduction of the ratio $t_{\mathrm{So}} / U$. As $U$ approaches $U \simeq 2.2 t$, the magnetic anisotropy overshoots because the bulk becomes magnetic as well.

We now discuss the physical effects not covered within our two main approximations, namely, treating the interactions at the mean field level and ignoring the Rashba spin-orbit term. In one dimension, collective spin fluctuations are expected to destroy the infinitely long-range order described by mean field theory. Still, for ribbons shorter than the spin correlation length $\xi(T)$, the mean field theory provides a fair description, very much like density functional theory describes properly the magnetization of clusters and nanomagnets. The spin correlation length $\xi(T)$ in graphene edges, calculated within the spin wave approximation and ignoring spin-orbit coupling [36], is $\xi \simeq$ $40 \AA$ for $T=75 \mathrm{~K}$. The magnetic anisotropy barrier to rotate the spins out of plane for the approximately 15 tin atoms of a zigzag stanene edge that long would be $\Delta \simeq 60 \mathrm{meV}$.

Inclusion of the Rashba coupling would have two consequences. First, lack of inversion symmetry would split the bands in the case of AF configurations. Second, it would break the in-plane $x y$ magnetic symmetry at the edges. 
In conclusion, we have studied the magnetic anisotropy of the ferromagnetic phase of the zigzag edges of graphene and graphenelike systems that can be described with a single orbital Hubbard model on a honeycomb lattice with spin-orbit coupling described with the Kane-Mele Hamiltonian. This includes a large class of two-dimensional crystals, such as silicene [6-8], germanene [9], stanene [10], iridates [11,12], and metal organic frameworks [13]. Since the electronic dispersion of the noninteracting edge states is fully determined by the spinorbit coupling, the resulting magnetic anisotropy effects, computed within a mean field approximation, turn out to be very strong: the system undergoes a metal-to-insulator transition when the magnetization is rotated out of the normal, and, for large values of $t_{\mathrm{SO}}$, the magnetic solutions are only stable for in-plane magnetization. For all values of the spin-orbit interaction, we find that the ground state energy occurs for in-plane magnetization and the edge states are gapped.

J.F. R. acknowledges financial support by MEC-Spain (FIS2010-21883-C02-01) and Generalitat Valenciana (ACOMP/2010/070), Prometeo. This work has been financially supported in part by FEDER funds. We acknowledge financial support by Marie-Curie-ITN 607904SPINOGRAPH.

* On leave from Departamento de Física Aplicada, Universidad de Alicante, San Vicente del Raspeig, 03690, Spain.

[1] J. M. D. Coey, Magnetism and Magnetic Materials (Cambridge University Press, New York, 2010).

[2] A. Smogunov, A. D. Corso, A. Delin, R. Weht, and E. Tosatti, Nat. Nanotechnol. 3, 22 (2008).

[3] A. N. Núñez and J. Fernández-Rossier, Solid State Commun. 152, 403 (2012).

[4] K. Nakada, M. Fujita, G. Dresselhaus, and M. S. Dresselhaus, Phys. Rev. B 54, 17954 (1996).

[5] C. L. Kane and E. J. Mele, Phys. Rev. Lett. 95, 226801 (2005).

[6] P. Vogt, P. De Padova, C. Quaresima, J. Avila, E. Frantzeskakis, M. C. Asensio, A. Resta, B. Ealet, and G. Le Lay, Phys. Rev. Lett. 108, 155501 (2012).

[7] A. Fleurence, R. Friedlein, T. Ozaki, H. Kawai, Y. Wang, and Y. Yamada-Takamura, Phys. Rev. Lett. 108, 245501 (2012).

[8] C. Xu, G. Luo, Q. Liu, J. Zheng, Z. Zhang, S. Nagase, Z. Gao, and J. Lu, Nanoscale 4, 3111 (2012).

[9] M. Houssa, G. Pourtois, V. V. Afanasev, and A. Stesmans, Appl. Phys. Lett. 96, 082111 (2010).
[10] Y. Xu, B. Yan, H.-J. Zhang, J. Wang, G. Xu, P. Tang, W. Duan, and S.-C. Zhang, Phys. Rev. Lett. 111, 136804 (2013).

[11] A. Shitade, H. Katsura, J. Kuneš, X.-L. Qi, S.-C. Zhang, and N. Nagaosa, Phys. Rev. Lett. 102, 256403 (2009).

[12] J. L. Lado, V. Pardo, and D. Baldomir, Phys. Rev. B 88, 155119 (2013).

[13] Z.F. Wang, Z. Liu, and F. Liu, Nat. Commun. 4, 1471 (2013).

[14] L. Brey and H. A. Fertig, Phys. Rev. B 73, 235411 (2006).

[15] K. K. Gomes, W. Mar, W. Ko, F. Guinea, and H. C. Manoharan, Nature (London) 483, 306 (2012).

[16] M. Polini, F. Guinea, M. Lewenstein, H. C. Manoharan, and V. Pellegrini, Nat. Nanotechnol. 8, 625 (2013).

[17] M. Fujita, K. Wakabayashi, K. Nakada, and K. Kusakabe, J. Phys. Soc. Jpn. 65, 1920 (1996).

[18] Y.-W. Son, M. L. Cohen, and S. G. Louie, Phys. Rev. Lett. 97, 216803 (2006).

[19] J. Fernández-Rossier and J. J. Palacios, Phys. Rev. Lett. 99, 177204 (2007).

[20] J. Fernández-Rossier, Phys. Rev. B 77, 075430 (2008).

[21] S. Rachel and K. Le Hur, Phys. Rev. B 82, 075106 (2010).

[22] D. Soriano and J. Fernández-Rossier, Phys. Rev. B 82, 161302 (2010).

[23] F. D. M. Haldane, Phys. Rev. Lett. 61, 2015 (1988).

[24] M. Laubach, J. Reuther, R. Thomale, and S. Rachel, arXiv:1312.2934.

[25] F. Geissler, J. C. Budich, and B. Trauzettel, New J. Phys. 15, 085030 (2013).

[26] C.-C. Liu, H. Jiang, and Y. Yao, Phys. Rev. B 84, 195430 (2011).

[27] See Supplemental Material at http://link.aps.org/ supplemental/10.1103/PhysRevLett.113.027203, which includes Ref. [28], for details of the collinear mean field ansatz and the anisotropy effect in chiral edges.

[28] D. Gonsalbez-Martinez, D. Soriano, J. J. Palacios, and J. Fernandez-Rossier, Solid State Commun. 151, 1075 (2011).

[29] H. Min, J. E. Hill, N. A. Sinitsyn, B. R. Sahu, L. Kleinman, and A. H. MacDonald, Phys. Rev. B 74, 165310 (2006).

[30] Y. Yao, F. Ye, X.-L. Qi, S.-C. Zhang, and Z. Fang, Phys. Rev. B 75, 041401 (2007).

[31] S. Konschuh, M. Gmitra, and J. Fabian, Phys. Rev. B 82, 245412 (2010).

[32] C.-C. Liu, W. Feng, and Y. Yao, Phys. Rev. Lett. 107, 076802 (2011).

[33] C. Tao, L. Jiao, O. V. Yazyev, Y.-C. Chen, J. Feng, X. Zhang, R. B. Capaz, J. M. Tour, A. Zettl, S. G. Louie et al., Nat. Phys. 7, 616 (2011).

[34] X. Zhang, O. V. Yazyev, J. Feng, L. Xie, C. Tao, Y.-C. Chen, L. Jiao, Z. Pedramrazi, A. Zettl, S. G. Louie et al., ACS Nano 7, 198 (2013).

[35] S. Rachel and M. Ezawa, Phys. Rev. B 89, 195303 (2014).

[36] O. V. Yazyev and M. I. Katsnelson, Phys. Rev. Lett. 100, 047209 (2008). 